\title{
The Spectrum Series: Grappling with the Overlap between Alzheimer's and Parkinson's Diseases
}

\author{
9th International Conference on Alzheimer's and Parkinson's Diseases, 11-15 March 2009, Prague, \\ Czech Republic
}

Gabrielle Strobel

Alzheimer Research Forum

\section{SPECTRUM OF NEURODEGENERATION COMES TO THE FORE}

28 May 2009. Sometimes in science, a concept lingers unattended in the collective back of researchers' minds for years; they sense there is something important to it but are not ready to grasp it head-on. Then something changes and, voila, the concept moves front and center. Such was the case with the issue of overlap in the major neurodegenerative diseases at the 9th International Conference AD/PD held 11-15 March in the Czech capital city of Prague. Previous AD/PD conferences had reflected the de-facto separation in the daily work of most scientists and clinicians in this field. Even though they spent days under the same roof, by and large the movement disorder people went to one meeting and the dementia people to another. But Prague was different. Rather than being treated as an inconvenient side issue that blurred boundaries, the extensive and multitudinous overlap between Alzheimer and Parkinson diseases (and also the frontotemporal dementias) was the focus of presentations and hallway discussion. Much of the buzz about this topic had spilled over into Prague from a preceding workshop in the former Royal German city of Kassel that had focused on dementia with Lewy bodies (DLB) and Parkinson disease dementia (PDD). These are two underappreciated conditions that occupy a large area of overlap between AD and PD. From 8-10 March, co-organizer Brit Mollenhauer of the Paracelsus-Elena-Klinik in Kassel had convened a group of investigators around the goals of sharing the latest insight and hammering out a research agenda to speed progress and fight for recognition of these betwixt diseases.

Once the overlap between two neurodegenerative diseases, not their differences, becomes the center of attention, the story changes in many ways, scientists said. Individual cases are comfortably seen as falling on a spectrum rather than having to fit into this box or that, and the view of the diagnosing physician changes such that (s)he expects and accommodates large numbers of mixed cases. Perhaps most importantly, the search for protein-based biomarkers - from fluid biochemistry to imaging - to tease out which underlying proteins drive a given person's disease, assumes paramount importance.

Traditionally, neurodegenerative diseases have been classified clinically, as physicians grouped them into boxes based on the preponderance of signs their patient presented - movement abnormalities in PD, cognitive deficits in AD at its simplest. Separately, pathologists described postmortem brain abnormalities and tried to match them up with symptoms. That clinicopathological pairing is descriptive, and it has been refined in recent years. But in reality, the pathology, seen years after diagnosis, often poorly matches the clinical diagnosis a patient had received. Or it even calls the diagnosis into question when, for example, a patient 
diagnosed as having AD turns out to have had extensive $\alpha$-synuclein but little tau pathology in the brain.

"There has been a conceptual shift in neurodegeneration research. Until recently the focus was on factors that distinguish between these disorders. But now we recognize that there is extensive overlap at the pathological level and the clinical level, which does not fully match the genetics. So it is difficult to distinguish cleanly by means of clinical, pathological, or genetic determination alone," said Kristel Sleegers of VIB in Belgium.

As science advances, the diagnosis of neurodegenerative diseases beyond AD itself will become increasingly molecular in an effort to pin down the pathogenic proteins that drive an individual person's disease rather than focus primarily on the symptoms. "In time, we will shift away from relying on clinical categorization to make diagnoses," said James Galvin of Washington University, St. Louis. "We will make protein diagnoses. The way to get there is to understand underlying pathways and to develop a range of biomarkers."

The need for protein markers (and eventually also RNA- and lipid-based markers) as sorting tools is evident from even a cursory look at how varied these diseases can be. For example, scientists are realizing that what looks like a single clinical entity has multiple causes. To quote but one emerging example, new studies of PD patients are fingering the gene for Gaucher disease, as well as a high-expression variant of the protein tau. Vice versa, a single pathogenic mutation, for example, in the gene progranulin, has been reported to manifest itself in the form of frontotemporal dementia, $\mathrm{PD}$, or even $\mathrm{AD}$ in affected members of one and the same family.

Confused already? Read on; there's more. DLB and PDD define a spectrum going from $\mathrm{AD}$ to PD that hinges on the specifics of aggregation of amyloid- $\beta$ $(\mathrm{A} \beta)$, tau, and $\alpha$-synuclein, but it is not the only spectrum at play. There is also a spectrum going from frontotemporal dementias to amyotrophic lateral sclerosis that hinges on loss of progranulin protein and accumulation of ubiquitin and TAR DNA-binding protein 43 (TDP43). Deposits of the latter have been reported in a significant fraction of AD cases, even, though whether they are mechanistically important to disease is entirely unclear at this point. And, of course, it matters greatly where a pathologic protein acts. For example, of the wide range of disorders blamed on $\alpha$-synuclein the Lewy body diseases - one particularly severe one called multiple system atrophy stands out by exhibiting this pathology primarily in oligodendroglia, not neu- rons. Yet another spectrum talks of tauopathy in parkinsonism. The number of invoked spectra conjure up a mental image less of a linear continuum between, say, blue and green, but more of a color wheel. Scientists readily agree that there are many more examples for heterogeneity at the clinical, pathological, and genetic levels.

"People are talking in terms of spectra now because the pure forms of these diseases are less common than we used to think, and the overlap is incredibly large," said Galvin. "Once you focus on patients in the overlap, you see that they are different from those with the pure forms of disease. That is why molecular diagnoses are going to be critical for this field."

Molecular diagnoses will require fluid or imaging markers based on individual proteins that drive disease either alone or in various combinations. The range of candidates is expanding well beyond the known ones such as $\mathrm{A} \beta$, tau, to now include $\alpha$-synuclein, progranulin, TDP43, glucocerebrosidase, and brain imaging based on neurotransmitter transporters. Such tests are being aggressively pursued in different labs.

Besides offering a more precise diagnosis, such tests may help scientists define what molecular interactions underlie yet another phenomenon scientists emphasized both in Kassel and in Prague - namely that when two of these proteins go awry in one person, they tend to heat up the pathogenic process and worsen the resulting clinical disease in a given person. "There's an emerging realization that whenever two pathologies occur together, they accelerate disease," said Michael Schlossmacher of the University of Ottawa, Canada. This, in turn, has created interest in studying possible links between the underlying proteins at the monomeric and oligomeric level.

The esteemed reader trying to keep track of these blurring boundaries might take solace in remembering that this added layer of complexity comes on top of a much simpler, underlying rule that has found wide acceptance across the field of neurodegeneration. It is that for the established, major proteins causing neurodegeneration when they aggregate - $\mathrm{A} \beta /$ amyloidoses, $\alpha$ synuclein/synucleinopathies, tau/tauopathies, prp/ prion diseases - mutations or duplication of the gene cause familial forms, whereas overproduction alleles raise the risk of sporadic forms. "This blindingly simple categorization is true for all of these diseases. The more you make of these proteins, the earlier you get the disease," John Hardy of University College, London, UK, said in Prague. And as a possible yang to this yin, the opposite trend is just beginning to emerge for progranulin 
and perhaps even the newest neurodegenerative disease protein, glucocerebrosidase. There, early indications are that disease risk goes up the less a person makes of the protein.

\section{ET TU, BRUTE? PARKINSON'S GWAS FINGERS TAU NEXT TO $\alpha$-SYNUCLEIN}

29 May 2009. At the 9th International Conference AD/PD, held this past March in Prague, Thomas Gasser of the Hertie Institute in Tuebingen, Germany, presented data that illustrated two emerging concepts in neurodegenerative disease research. First, the same gene can cause disease in various guises - as an inherited mutation in some familial cases or as a risk variant in some sporadic cases. Second, the overlap between related neurodegenerative diseases is tremendously large and varied. The overlap cuts across the clinical, pathological, and genetics levels, and tau rears its head again and again.

Gasser presented the results of the largest genomewide association study (GWAS) performed to date on PD patient samples. In PD, geneticists in the past decade have assembled a list of some 15 genetic loci. Even so, the large majority of the genetic variance underlying the total burden of PD remains unexplained. The best-understood gene, $\alpha$-synuclein, gives rise to rare familial PD when mutated, duplicated, or triplicated. However, at the level of pathology, its Lewy body signature is much more widespread than that. Indeed, even beyond PD, $\alpha$-synuclein pathology shows up in a range of other $\alpha$-synucleinopathies. The genetic contribution of $\alpha$-synuclein alleles to that larger burden of neurodegenerative disease is largely unknown.

The present GWAS represents a collaboration of an international PD Genetics consortium with Andrew Singleton at the National Institutes of Health in Bethesda, Maryland, and additional groups elsewhere, Gasser said. The scientists studied 463,000 quality-controlled SNPs per sample - a huge number as such studies go in some 1,700 quality-controlled (qc'd) cases and 4,000 qc'd controls from mostly Caucasian U.S. and German samples in a first stage, and then analyzed the 384 top SNPs from this stage in a second sample of 3,500 cases and 4,200 controls. "This gave us good power to detect common risk factors conferring modest effects," Gasser said.

Which genes survived statistical correction to remain above the Bonferroni line? Only two did - not LRRK2, not parkin, PINK1, or DJ1. $\alpha$-synuclein and tau stood out in both stages of the GWAS, Gasser reported. For $\alpha$-synuclein the result was somewhat expected. It further cemented the relationship in certain key genes between pathogenic mutations, duplication, or triplication causing familial and risk alleles promoting sporadic forms of PD. In contrast, tau's appearance at first glance may seem an astonishing betrayal by a protein implicated in AD and FTD, not primarily PD. But it should not, noted other researchers. For example, in some cases of PD, tau pathology is readily apparent, said Michael Schlossmacher of the University of Ottawa, Canada. Suspicions about tau playing a role in the pathogenesis of PD arose years ago. Since then, smaller genetic studies have implicated tau in PD in such a way that a low-expression variant appears to be mildly protective. In the present GWAS, tau's genetic effect may have been mediated through mRNA expression levels, Gasser said. And the idea is catching on. German researchers recently proposed a spectrum of tauopathy with parkinsonism, and yet other scientists have blamed certain tau variants for the dementia that develops in many patients with advanced PD. Greetings from the overlap - again.

"In most GWASs of these neurodegenerative diseases, tau comes up. It is not clear what it does, but one thought is that here it could act as a switch to augment $\alpha$-synuclein pathology," commented Douglas Galasko of the University of San Diego, California.

Indeed an old finding from familial AD brought up this connection between tau and $\alpha$-synuclein, when researchers observed that people who had inherited an autosomal-dominant presenilin or $\mathrm{A} \beta \mathrm{PP}$ mutation at autopsy turn out, besides the expected plaques and tangles, to also have had Lewy body pathology in their brains. Researchers increasingly agree that $\mathrm{A} \beta \mathrm{PP}$ or presenilin lies upstream of tau in the AD disease pathway, but tau then appears to somehow fire up $\alpha$ synuclein, as well. What exactly goes on between the two, or whether the pathways are separate, is anyone's guess at this point, scientists said.

Beyond pointing an accusing finger at tau, the new GWAS presented by Gasser confirms the association of $\alpha$-synuclein variants and sporadic PD found in previous, smaller studies. The odds ratio for $\alpha$-synuclein computes to 1.4 for carriers of one risk allele and to 1.9 for carriers of two; together this could account for some 9 percent of the population risk for PD, Gasser said in his talk. The idea is that certain risk alleles increase $\alpha$-synuclein protein levels. How that might happen, however, remains unclear as the mechanism appears to be more complicated than simple differences in $\alpha$ - 
synuclein mRNA levels, according to ongoing research in the laboratory of John Hardy at University College, London.

A flip side to this overlap between neurodegenerative diseases (i.e., one clinical entity - different genes) is that one gene can give rise to different clinical pictures. The new GWAS sheds light on this, too. Consider multiple system atrophy (MSA). People with this severe and mysterious disease deteriorate rapidly from parkinsonism or ataxia (or both), and from concomitant failure of their autonomic nervous system. Their brains show $\alpha$-synuclein pathology mainly in glial cells. MSA appears entirely sporadic, as no familial cases have been described as yet. But it has enough in common with PD that Gasser and colleagues decided to check whether any of the 384 top SNPs from the PD GWAS were associated with MSA. This second genetic study, as well, was large for such a rare disease, first testing the SNPs in 413 cases and nearly 4,000 controls and then replicating the top 10 SNPs from that stage in a second, independent cohort. Fifteen different institutions in Europe and the U.S. contributed samples to this study, Gasser noted. $\alpha$-synuclein emerged as the only MSA risk gene to survive replication and statistical correction. It also stood through further replication in a third sample of 100 pathology-confirmed cases from the Institute of Neurology in London, UK. For MSA, the odds ratio of carrying a homozygous $\alpha$-synuclein risk allele may be as high as 6.2, Gasser showed.

Overall, then, this GWAS drove home the message that $\alpha$-synuclein alleles that lead to higher expression or protein levels can increase a person's risk for PD and at least one other $\alpha$-synucleinopathy, MSA. Tau, too, plays a genetic role in Lewy body diseases. Gasser estimated that taken together, the genes for $\alpha$-synuclein and tau may account for a fifth of Parkinson disease cases.

Questions after his talk revolved around the notion of whether two pathologies, when they occur together, might fire each other up to accelerate disease. The GWAS does not directly address this because the tau and $\alpha$-synuclein risk variants were independent of each other. But the question deserves study, Gasser said. To many neurogeneticists, tau's hand on the tiller of Lewy body diseases may come as no surprise. Tau has been placed at the center of another neurodegenerative disease spectrum before, namely that from AD to FTD. And just this month, British researchers reported having spotted it even further afield, in an aggressive form of multiple sclerosis.

\section{NEITHER FISH NOR FOWL - DEMENTIA WITH LEWY BODIES OFTEN MISSED}

1 June 2009. Perhaps the biggest, and quintessential, representative of a spectrum neurodegenerative disease is dementia with Lewy bodies (DLB). By some counts, this disease is the second most common form of dementia after $\mathrm{AD}$, with patient estimates for its various forms ranging between one and two million in the U.S. All the same, DLB has struggled for recognition and research dollars, being squeezed uncomfortably between its two large neighbors AD and PD. "DLB research is an unappreciated field," said Brit Mollenhauer of the Paracelsus-Elena-Klinik in Kassel, Germany.

DLB is a double whammy of a disease. People with DLB have behavioral and memory problems as in AD and, to a varying extent, also suffer motor symptoms as seen in PD. However, the cognitive symptoms of people with DLB tend to fluctuate frequently, their motor symptoms are milder than in PD, and DLB patients often have vivid visual hallucinations and particular visuospatial deficits. In short, DLB is neither AD nor $\mathrm{PD}$, and yet defining its distinct identity has been a challenge. At the 9th International Conference AD/PD held last March in Prague, ample discussion about DLB resonated from an immediately preceding workshop on this disease and its cousin, Parkinson disease dementia (PDD). Co-organized by Mollenhauer and Richard Dodel, the workshop tried to put DLB more firmly on the research map.

"Scientists who study DLB think it is a very important disease," said James Galvin of Washington University, St. Louis. "As a set of independent groups, we and others have worked to increase its face time in the dementia world. We fight a battle because given the limited time and resources funders and reviewers have available to cover related conditions, DLB tends to get the short end of the stick."

Why is that? Part of the reason is that carving distinct disease categories out of a continuum of symptoms and pathologies is inherently arbitrary. Part of it is that multiple labels being advanced by different investigators for multiple similar variants have not helped the branding. In Prague, several scientists noted that if its awkward name was part of DLB's identity problem, one solution might be to name it after Kenji Kosaka of Houyuu Hospital in Yokohama, Japan. "Kosaka himself called it a different name, but he really described the clinico-pathological entity that we nowadays diagnose as DLB," said Michael Schlossmacher of Ottawa University, Canada. "Alzheimer disease, Parkin- 
son disease, Kosaka disease would sound consistent and recognizable to me."

The Kassel workshop was the latest in a series of small international meetings by a consortium of groups interested in DLB and PDD. In 1995, researchers led by Ian McKeith of Newcastle General Hospital met in Newcastle-upon-Tyne, UK, to hammer out consensus diagnostic criteria. This spurred diagnosis in specialty settings and provided a basis for gathering incidence and prevalence data there. But the consensus criteria have not widely penetrated community geriatric, neurology, or primary care settings where many patients are still seen, and both the rate and accuracy of DLB diagnosis remain low. "We are very bad at diagnosing DLB. Up to half of cases diagnosed as DLB turn out at autopsy to have had AD. Misdiagnosis of DLB as AD occurs, as well," said David Brooks of Imperial College, London.

This has serious consequences. The U.S. Food and Drug Administration, for example, does not formally recognize DLB as a distinct disorder. The agency has a point, Galvin concedes. "They ask: Is it AD? Is it PD? What exactly is it? They ask: Do doctors recognize DLB as separate? No? Then how can you run drug trials, and how can you get doctors to prescribe a future DLB drug?" Galvin said.

With their series of DLB/PDD workshops, McKeith's and other groups aim to forge a common research agenda that can move their nascent field forward in a concerted way. Subsequent to Newcastle, workshops in the Dutch city of Amsterdam and Yokohama, Japan, continued the effort; and this year's gathering in Kassel was to lead up to an official centenary workshop in 2012 that will celebrate Frederick Henry Lewey's first description of Lewy bodies in 1912. (Born in Berlin as Fritz Heinrich Lewy, this German Jewish neurologistcum-pathologist in his early years worked with Emil Kraepelin and Alois Alzheimer, but was forced in 1933 emigrate to England and then the U.S.) This year's workshop designated working groups for biomarkers and for clinical trials, said Mollenhauer. It also included representatives from national DLB societies and patient groups in an effort to help these lay groups beef up their operations such that they can become larger funders of research and lobby for federal funding and recognition, akin to what the Alzheimer's Association has accomplished for its disease.

Below are some of the main problems, and points of consensus, about DLB from the Kassel and Prague meetings. Part of the reason why more research groups have not taken up focused study of DLB is its com- plexity. DLB is marked by overlap with AD and PD on the clinical level and on the postmortem pathology level. But clinic and pathology do not match up to a clean picture, leaving the scientist to juggle a welter of descriptive facts that for many fail to "gel" into a tangible entity. Eventually, the solution to this problem will come with new biomarker-driven diagnoses, but even in the meantime, clinicopathological correlations have come a long way, the Kassel workshop made clear.

Clinicians agree that people with mixed pathologies suffer faster and more severe disease. Pure Lewy body pathology exists in 10 to 20 percent of cases with clinical DLB, but the majority of patients also have amyloid pathology and many have tangles to varying degrees, as well. Some even have aggregates of TDP43, though whether that is functionally important is not known yet. It is beyond dispute, however, that mixed pathologies compound each other. Several groups have found that when they looked at postmortem pathology and compared the clinical and cognitive course of the respective patients during their lives, the mixed cases always performed more poorly and progressed faster. Time to nursing home placement, time to death, visuospatial deterioration - whatever the outcome, the mixed cases fared worse. "Pure and mixed clearly are different diseases," Galvin said.

In the year 2005, the 1996 DLB consensus criteria were revised to focus on the spectrum of Lewy body disorders and to explain more clearly the links between symptoms and pathology. The clinical aspects that set DLB apart from AD, for example, were ascribed to $\alpha$-synuclein pathology. In a nutshell, this is what the criteria said: DLB and AD share amyloid pathology; people with DLB have $\alpha$-synuclein pathology, as well, but generally few neurofibrillary tangles. The more tangles a person has, the more their clinical picture overlaps with AD; the fewer tangles they have, the more it diverges from AD.

In the past four years, several groups have further sharpened the cognitive profile of DLB. The goal is to separate DLB not just from AD but also from PDD, where a patient first has PD for some years and then develops dementing symptoms. For example, David Salmon of the University of California, San Diego, showed in Kassel that despite some general similarities between DLB and PDD, PDD is marked by deficits in psychomotor speed and attention, which probably arise as $\alpha$-synuclein pathology spreads from the brainstem via limbic structures and across the cortex. As long as $\alpha$-synuclein pathology in the cortex remains mild, PD patients tend to stay cognitively intact. DLB 
has in common with AD verbal memory deficits driven by these diseases' shared amyloid and tau pathologies. That is another point of distinction from PDD. But DLB differs from AD by showing pronounced deficits in visuospatial and executive function that Salmon attributes to a unique combination of cortical amyloid and $\alpha$-synuclein pathology. These differences are useful early on in disease; in late stages the clinical picture of these diseases increasingly merges. Overall, visuospatial tests seemed the most useful for picking out patients with DLB, and these tended to be the people most likely to deteriorate quickly. They also tended to be the ones who suffered visual hallucinations. Beyond these means, disentangling in more detail which clinical features arise from AD pathology and which ones from $\alpha$-synuclein pathology will require $\alpha$-synuclein-based and AD pathology-based biomarkers.

For his part, Galvin and colleagues recently developed cognitive profiles that distinguish $\mathrm{AD}$ from healthy brain aging. Compared against these profiles, a group of DLB patients performed quite differently from $\mathrm{AD}$, as well. Combining these cognitive data with clinical and amyloid imaging data, Galvin has devised a clinical risk score that detects LBD. "This gives us a good separation. We find that some people who were clinically diagnosed with AD turn out to probably have DLB," Galvin said. In the laboratory, his group is working on cerebrospinal $\alpha$-synuclein detection to support this prediction. The goal is to be able eventually to define preclinical DLB. This would work such that a person who is positive for brain amyloid by PET imaging but is cognitively normal could receive CSF biochemistry testing for $\mathrm{A} \beta /$ tau and for $\alpha$-synuclein to determine whether (s)he will likely go on to develop AD or DLB. "We have great confidence in predicting $\mathrm{AD}$ based on the PIB/CSF $\mathrm{A} \beta$-tau combination. We want to achieve the same confidence to predict DLB," Galvin said.

\section{LIKE DLB, LIKE AD - DO OLIGOMERS STIR UP THE TROUBLE?}

2 June 2009. Current work on distinguishing Alzheimer disease from its cousin dementia with Lewy bodies (DLB) has underscored one intriguing similarity between the two. In DLB, researchers increasingly note that many people, indeed up to half in some patient series, remain neurologically intact despite having abundant Lewy body pathology in their brains. In AD, florid amyloid pathology in the brains of people who died cognitively normal has for years sustained doubt about the amyloid hypothesis. In DLB now as formerly in $\mathrm{AD}$, the pathologic observation raises questions about whether Lewy bodies are toxic or even relatively protective compared with even more toxic oligomeric aggregates of $\alpha$-synuclein that remain invisible to the stains typically used on brain slices.

At the Kassel workshop preceding the 9th International Conference AD/PD, Laura Parkkinen of the Institute of Neurology, London, UK, broached this issue in a clinico-pathological talk. Similarly, Walther Schulz-Schaeffer of the University of Goettingen, Germany, proposed that not Lewy bodies, but smaller $\alpha$ synuclein aggregates at synapses, are the real culprits. And at AD/PD in Prague, Maria-Grazia Spillantini of Cambridge University, UK, previewed data from a new mouse model of $\alpha$-synucleinopathy that pinned early pathogenic changes on mislocalization of monomeric $\alpha$-synuclein within presynaptic terminals. Spillantini proposed that the neurons seen laden with Lewy bodies in autopsy tissue might represent those cells that were the latest to have gotten sick, i.e., that have withstood a disease process driven by smaller assemblies.

Mice are also the model of choice to try to understand whether different pathogenic proteins interact, perhaps as oligomers, before they form their signature microscopic deposits. Eliezer Masliah of the University of California, San Diego, has for some time explored molecular interactions between $\mathrm{A} \beta$ and $\alpha$-synuclein, showing first that $\mathrm{A} \beta$ potentiates the deposition of $\alpha$ synuclein in transgenic mice and more recently that these two proteins can form mixed ring-like oligomers in membranes. In Kassel and in Prague, Masliah expanded on the theme. He introduced several different unpublished mouse systems that combine transgenic lines and lentiviral injection. Together, these build a body of data suggesting that $\mathrm{A} \beta_{42}$ promotes $\alpha$ synuclein aggregation, worsens learning deficits, and can drive neurodegeneration in these mixed models. This happens regardless of whether $\mathrm{A} \beta \mathrm{PP}$ is added to an $\alpha$-synuclein transgenic background or $\alpha$-synuclein is added to an $\mathrm{A} \beta \mathrm{PP}$-transgenic background.

Most likely, $\mathrm{A} \beta$ is upstream of $\alpha$-synuclein, researchers agreed. This creates a parallel with older $\mathrm{AD}$ research placing $\mathrm{A} \beta$ upstream of tau, and it puts $\alpha$-synuclein and tau on a par in a sense. Expressed in mice, the mutant human tau that causes frontotemporal dementia leads to tangles and a behavioral phenotype mostly in the spinal cord, but when these mice crossbreed with $\mathrm{A} \beta \mathrm{PP}$ transgenic mice, the $\mathrm{A} \beta$ in the resulting offspring greatly amplifies tau pathology in the 
cortex. The idea is that, similarly, the co-occurrence of $\mathrm{A} \beta$ might help $\alpha$-synuclein pathology spread in the human brain.

"The idea of one pathology augmenting another is accepted in AD, and now comes up in DLB, as well," noted John Hardy of University College, London, UK. Other scientists agreed that amyloid pathology probably deposits first in people, before inciting either tau or $\alpha$-synuclein pathology. More than two pathogenic proteins can be at play, as some scientists suspect tau heating up $\alpha$-synuclein pathology downstream of amyloid. "In the mixed pathologies, we know that amyloid deposits first. Then there occurs some unknown step that we need to understand much better," said Galvin.

This point drew wide notice in Prague. "Broadly, the idea that one of those proteins can influence aggregation of another is gaining prominence," said Charles Glabe of University of California, Irvine, who mentioned collaborative work with a German group indicating that therapeutic removal of amyloid can draw down $\alpha$-synuclein inside cells. "The big question is how that happens, whether directly at the membrane or through activating autophagy."

Interaction between $\mathrm{A} \beta$ and $\alpha$-synuclein might imply that upstream (read anti-amyloid) therapies might benefit downstream $\alpha$-synuclein pathology (read DLB patients), as well. Masliah showed data suggesting that anti-A $\beta$ immunotherapies treat synucleinopathy and attendant functional deficits quite nicely in transgenic mice. Alas, the known trials and tribulations of translating mouse treatments to humans apply. Other scientists cautioned that diseases marked in large part by pathologies downstream of $\mathrm{A} \beta$ amyloid might at some point become independent of that amyloid once disease is established, such that removing the initial offender no longer helps the patient very much because it leaves in place an active tauopathy or synucleinopathy.

Antibodies against $\alpha$-synuclein are under construction in Masliah's laboratory. In Prague, Brian Spencer in Masliah's lab presented a poster showing a lentivirus single-chain antibody against $\alpha$-synuclein oligomers. When injected into the brain of $\alpha$-synuclein transgenic mice, the antibody rescued neurodegeneration in these animals. The mechanism, Masliah believes, is not so much microglial clearance but activation of the autophagy pathway of protein degradation. This, if it could be revved up safely, might just offer a new therapy development avenue to pursue against both $\mathrm{AD}$ and DLB.

\section{ORDNUNG, PLEASE - CAN BIOMARKERS TAME A BEWILDERING OVERLAP?}

3 June 2009. Faced with a complicated landscape of mixed disease at all levels of observation, scientists at the 9th International Conference AD/PD last March in Prague made one point abundantly clear. Even as the recognition that neurodegeneration occurs on a spectrum is gaining prominence throughout the field, tangible progress in dealing with spectrum diseases will remain limited until the field comes up with more and better biomarkers of their component proteins. "It is apparent that the next phase of refinements to clinical classification will need to incorporate the use of biological markers of underlying disease process, since clinical presentation alone is an unreliable witness of pathology," is how Ian McKeith of Newcastle General Hospital in Newcastle upon Tyne, put it in his opening abstract to a workshop in Kassel, Germany, that preceded AD/PD. The same challenge applies to the spectrum of progranulin diseases. Protein-based markers could address the common problem of misdiagnosis of DLB. In clinical testing, biomarkers could avoid several problems, for example that of trials recruiting patients with different underlying diseases into a single treatment group, or the problem of enrolling patients with simmering preclinical disease into control groups, or of enrolling a person with, e.g., a progranulin-driven dementia into an anti-amyloid drug trial.

What, then, do scientists have in hand? In short, they have candidates in various stages of refinement but no officially validated winners yet. This conference story will summarize some of the imaging markers currently under study for use in diseases of the $\alpha$-synuclein and progranulin spectrum. The next story will summarize fluid markers.

First, brain imaging. And first, the bad news. $\mathrm{Nu}-$ merous groups are working on contrast agents and radioligands that would find and label aggregates of $\alpha$ synuclein and also tau, but no one appears to have a candidate ready for trial in humans. Michael Pontecorvo of the molecular imaging company AVID Radiopharmaceuticals Inc. is usually a fluent speaker with the polish of a company pitchman. But when asked where things stood on a PET ligand for tau, all he could say was, "We are not close." For a-synuclein? "Working on it." How about A $\beta$ oligomers? "Nope. . . I wish."

Pontecorvo was more loquacious about dopamine transporter imaging. SPECT scans using ligands for this molecule are already in routine clinical use to diagnose Parkinson disease. In Prague, Pontecorvo pre- 
sented phase 1 data on an experimental PET ligand for essentially the same purpose. AVID sees advantages because the new agent labels a presymptomatic vesicular monoamine transporter, VMAT2. Its levels decrease with disease but are not up-or downregulated in response to L-Dopa treatment, Pontecorvo said. Called $18 \mathrm{~F}-\mathrm{AV}-133$ at present, the new ligand enters and leaves the brain rapidly, meaning it could be imaged sooner after the patient receives the injection and would shorten the time the patient has to lie still in the scanner. In a small pilot study, AV-133 distinguished AD from DLB, Pontecorvo said in Prague. PD and DLB patients both showed a reduction in requisite brain areas, whereas participants even with fairly advanced AD looked like controls. The company also has an amyloid imaging ligand, AV-45 aka Florpiramine, which at present is in a Phase 3 trial and serves as a biomarker in some AD drug trials, though it has no peer-reviewed papers in the scientific literature. Avid hopes eventually to sell the dopamine transporter ligand and Florpiramine to support differential diagnosis along the spectrum going from AD, DLB, PDD, to PD. "You could scan the same person with both compounds on the same day in three to four hours," Pontecorvo said.

For his part, David Brooks, who works both at Hammersmith Hospital and for G.E. Healthcare, the commercial developer of Pittsburgh compound B (PIB), reviewed brain imaging approaches for this disease spectrum more broadly. Regarding dopamine transporters (DAT), Brooks cited an older study showing that DAT scans of the striatum distinguish DLB from AD during a person's life. Since then, postmortem follow up of people who had undergone DAT scans have shown that whenever the pathologist definitively diagnosed DLB, the person's DAT scan had been abnormal, whereas when the definitive diagnosis said AD the DAT scan had been normal. A phase 3 multicenter trial further validated this method.

By contrast, on a different method advanced for distinguishing DLB from AD, Brooks noted that his group was unable to reproduce previous data by others. That data had suggested that measuring atrophy in the medial temporal lobe might discriminate. This imaging method is more valuable for following disease progression, Brooks said.

Amyloid imaging can be one component of a DLB diagnosis, Brooks said. New $\mathrm{A} \beta$ radioligands are joining an increasingly competitive field. The latest is perhaps AstraZeneca's 11CAZD2184 compound, which Samuel Svensson debuted in Prague. These new compounds are just beginning to be tested on a broader scale. The older compound PIB ("older" meaning all of five years) has since 2004 been used at a growing number of independent institutions. It has by now generated a critical mass of data to indicate that, overall, a small majority of patients diagnosed with DLB have brain amyloid loads approaching those of people with AD, Brooks said.

A much smaller percentage of people diagnosed with PDD are PIB-positive. In contrast to DLB, which causes both motor and mental symptoms from the get-go, PDD is a dementia that develops when PD progresses and spreads outward from the nigrostriatal system. PET studies following the fate of dopaminergic and cholinergic neurons show that PDD manifests itself as neuron loss expands from the motor cortex to the parietal and frontal cortex. This causes both a dopaminergic and sweeping cholinergic loss. The former is responsible for increasing disability, the latter for cognitive decline, Brooks concluded. It is clear, however, that "in PDD, the dementia is not caused by amyloid," he added.

For the purpose of predicting whether a PD patient is likely to develop dementia in the next few years, FDG PET of neuronal activity in cortical areas of the brain appears helpful. Inflammation as imaged with the microglial activation marker 11C-PK11195 also precedes dementia in PD, Brooks said in Prague. Up to 80 percent of people with PD suffer this fate, but typically not before having lived with PD for a decade or more.

\section{STILL EARLY DAYS FOR $\alpha$-SYNUCLEIN FLUID MARKER}

4 June 2009. In AD research, well over a decade of intense research into fluid biomarker candidates has reached a point where a so-called "pathological signature" of $\mathrm{A} \beta$ and tau proteins is beginning to emerge from the 59-center Alzheimer's Disease Neuroimaging Study. This signature validates on a larger scale a number of earlier studies that had shown essentially the same thing. Coming, as it did, with ups and downs along the way, this search for a fluid test is guiding researchers who are working to develop similar markers for $\alpha$-synuclein and progranulin, two major proteins involved in many of the overlapping forms of dementia at issue in earlier parts of this news series. The 9th International Conference AD/PD, held last March in the Czech capital city of Prague, as well as an immediately preceding workshop on dementia with Lewy bodies (DLB) and Parkinson disease dementia (PDD) in 
Germany, showcased a rapidly growing field of groups who are racing to broaden the field. Here is a selection.

First, $\alpha$-synuclein. The three investigators who started fluid-based markers on this intraneuronal protein are Michael Schlossmacher, now at the University of Ottawa, Canada, Brit Mollenhauer, now at ParacelsusElena-Klinik in Kassel, who worked in Schlossmacher's former lab at Brigham and Women's Hospital in Boston, and Omar El-Agnaf, now at United Arab Emirates University in Al Ain, UAE. All three collaborated extensively, first to build ELISA assays and to show that these assays can quantify $\alpha$-synuclein in normal human cerebrospinal fluid (CSF), then to show that the CSF concentration of this protein normally declines with age and declines even further with Parkinson disease. A first cross-sectional study compared CSF $\alpha$ synuclein concentration in various patient groups, i.e., AD, DLB, PD, multiple system atrophy (MSA), and Creutzfeldt-Jakob disease (CJD) with controls. These studies found the lowest levels in PD and MSA, whereas AD and controls had similar and higher levels, and DLB lay in between. In CJD, $\alpha$-synuclein was curiously elevated, perhaps because the rapid cell death in this condition dumps this protein into the CSF so that it serves as a marker of degeneration in this situation, much as tau is viewed in AD.

However, the same difficulty that has dogged CSF measurements of $\mathrm{A} \beta$ since the beginning quickly caught up with $\alpha$-synuclein, too. Its concentration varies greatly from person to person, creating enough overlap that the test in its original form is unable to distinguish which group a given person falls into. While as a group, the values of people with PD always cluster at the bottom, any given person with $\mathrm{PD}$ might have a value higher than a control or an AD patient. Moreover, other research groups, using their own, different ELISAs, have been unable so far to replicate Mollenhauer and colleagues' result, calling into question whether $\alpha$-synuclein can serve as a robust diagnostic marker to distinguish between overlapping diseases. In Prague, debate centered on the different assays and antibodies different groups are using to measure $\alpha$-synuclein. Schlossmacher noted that his and collaborators' ELISA is extensively validated. Other scientists agreed that before a final word can be spoken, more tests in additional patient cohorts, independent replication, a comparison of methods, and exchange of antibodies are needed.

"Right now, many groups are trying to measure CSF $\alpha$-synuclein and are having a hard time seeing good separation between the groups. We, too, see a very narrow range of values," said James Galvin of Washington University, St. Louis, Missouri. "But that's no reason to be discouraged. It may just take more standardization of the steps and the right tools to get it down."

In Kassel, Mollenhauer presented new data on total CSF $\alpha$-synuclein measured in a separate cross-sectional cohort of clinically diagnosed patients. Again, PD and MSA lay at the bottom, AD and controls at the top, DLB in between. The overlap remained extensive, though expressing $\alpha$-synuclein concentration relative to total protein teased the groups apart somewhat. In Prague, Mollenhauer's poster of a separate series of 41 autopsyconfirmed cases showed that their CSF measurement matched the working diagnosis they had received during life.

On balance, then, the early days of $\alpha$-synuclein biomarker research have made clear that this protein can be directly measured in the CSF and peripheral blood, and, least with one assay, trends downward from controls to DLB and PD, Schlossmacher said. But besides technical collaboration to streamline protocols, much more scientific work remains to be done, he added. Challenges include understanding where exactly the CSF $\alpha$-synuclein comes from (the brain, the periphery, the choroid plexus could all contribute), what different species of $\alpha$-synuclein occur in CSF (truncated, fulllength, or modified), and which one of those best indicates disease. To see how these species change in the same person over time, Schlossmacher's and Mollenhauer's groups have begun longitudinal studies.

Galvin foresees a future where academic centers interested in earlier-stage clinical trials use a CSF assay for $\alpha$-synuclein to distinguish preclinical AD from preclinical DLB. An $\alpha$-synuclein imaging ligand is not on the horizon, but amyloid imaging is available and it shows a large fraction of non-demented elderly people who have brain amyloid and may turn out to be presymptomatic for either AD or DLB. Most DLB cases share A $\beta$ and $\alpha$-synuclein pathology; hence, an $\alpha$-synuclein fluid assay could conceivably flag amyloid-positive people who are at high risk for future DLB, much like combining amyloid imaging with $\mathrm{CSF} A \beta /$ tau measurement is predicting who will develop AD symptoms. Other groups are drilling deeper with $\mathrm{A} \beta$ biochemistry, measuring some of its truncated and oxidized forms to distinguish between $\mathrm{AD}$ and DLB.

"In our studies, we already have a number of people who are PIB positive and are not demented, but when you look at them with some of the biomarkers we are 
developing, some of these people are clustering with the DLB group. The idea is to be able to diagnose preclinical DLB," Galvin said. The Kassel meeting ended with the designation of a working group to hammer out a research path toward that goal, Mollenhauer wrote to ARF.

For his part, El-Agnaf has focused on measuring oligomers of $\alpha$-synuclein, initially in plasma and more recently in brain extracts. In Prague, he showed the results of a study looking for such oligomers in lysates prepared from postmortem brains of people who had suffered from DLB. As measured by a sandwich ELISA El-Agnaf developed with a commercial antibody that recognizes $\alpha$-synuclein aggregates but not monomers, these brains contained far higher concentrations of $\alpha$ synuclein oligomers than control or AD brains. The data showed less overlap between the groups, but no clear separation, either. Since then, the researchers used their ELISA on CSF samples and again found high levels. A final cohort of 60 samples from people with PD and controls displayed, again, a group difference but also a spread of the individual data points and overlap between the groups. Calculating the ratio of oligomeric $\alpha$-synuclein to total $\alpha$-synuclein improved the separation, El-Agnaf noted. "This is the first time we have been able to detect soluble oligomers from CSF in humans," El-Agnaf said in Prague, and here, too, the work of replication and broadening the effort is only just beginning.

Meanwhile, research underpinning the rationale for going after oligomeric $\alpha$-synuclein in body fluids is advancing in parallel. Here, too, Prague offered some news. For example, in the last talk of the AD/PD conference, Kostas Vekrellis of the Biomedical Research Foundation Academy of Athens, Greece, reported that secreted $\alpha$-synuclein oligomers are up to no good. Even though $\alpha$-synuclein is primarily a cytosolic protein, scientists know that cultured cells can release it. Cells also can take up external $\alpha$-synuclein, usually at their peril as they tend to die soon after, Vekrellis said.

Vekrellis investigated this apparent toxicity with lines of human neuroblastoma cells that can be induced to express wild-type $\alpha$-synuclein. Soluble monomeric and oligomeric $\alpha$-synuclein showed up in the conditioned medium from these cells. Using liquid chromatography-mass spectrometry proteomics and electron microscopy, Vekrellis and colleagues showed that the cells actively export $\alpha$-synuclein via an exosome pathway that itself depends on intracellular calcium. These cells sustained no harm from the $\alpha$-synuclein. But their conditioned medium, when squirted onto primary rat cortical neurons, killed those cells. A high-molecular-weight fraction of $\alpha$-synuclein species proved toxic. Medium depleted of $\alpha$-synuclein, or medium subjected to oligomer-busting compounds such as scylloinositol did not, pointing to oligomers as the active component. This new data invoke parallels with $\mathrm{A} \beta$ oligomers, which have been shown to impair synaptic activity and to damage cells, and whose sensitivity to scylloinositol has led to a Phase 2 trial.

\section{MEET PROGRANULIN, THE BIOMARKER - A SIMPLER STORY?}

5 June 2009. Scientists wrestling the complexities of $\alpha$-synuclein fluid biochemistry be forgiven for looking with some envy to a different protein of the neurodegenerative disease spectrum. At the 9th International Conference AD/PD, held last March in Prague, the field learned that crafting such a test for progranulin might actually be comparatively easy - incredible as that sounds in the field of neurodegeneration where generally speaking nothing is easy. Progranulin is the protein behind a sizable fraction of frontotemporal dementia and probably also a small, still-unknown fraction of cases diagnosed clinically as early-onset Alzheimer's or related disorders. In Prague, three independent groups presented results of their fledgling ELISA tests - one in serum, one in plasma, and one in cerebrospinal fluid. Incredible as it may seem to a biomarker field plagued by inconsistent findings on blood tests for other proteins such as $\mathrm{A} \beta$, all three groups reported the same overall result: it works just fine, thank you.

Progranulin surfaced independently in the laboratories of Christine van Broeckhoven at the VIBUniversity of Antwerp, Belgium, and of Michael Hutton, then at the Mayo Clinic Jacksonville, Florida, as the gene for the tau-negative form of frontotemporal dementia 17 (FTLD-U). This highly familial disease frequently strikes people younger than 65 . Since then, 66 pathogenic mutations in progranulin have turned up. Importantly, progranulin causes neurodegeneration by a different mechanism than $\mathrm{A} \beta$, tau, or $\alpha$-synuclein. Whereas these latter proteins are thought to become toxic as their concentration rises (i.e., the more protein, the earlier one gets sick), progranulin leads to neurodegeneration when there is not enough of it. With $\mathrm{A} \beta$, tau, and $\alpha$-synuclein, mutations that drastically increase expression cause familial early-onset disease, whereas risk alleles influence sporadic disease. In con- 
trast, the general theme emerging from progranulin genetics is that null mutations that slash protein levels in half cause familial FTLD-U, while milder missense mutations that cause a partial loss of function have a susceptibility role in $\mathrm{AD}$, amyotrophic lateral sclerosis, and perhaps PD, van Broeckhoven said in her talk in Prague.

Progranulin's different mechanism should translate into differences in diagnosis and treatment. The gene itself is plenty complicated, and the six different granulin proteins resulting from it have physiological functions throughout the body. But diagnosis might be straightforward. "We thought progranulin protein levels should be decreased in the blood of people with mutations that cause loss of function," Kristel Sleegers in van Broeckhoven's group said in her talk. Sleegers started with an ELISA against full-length progranulin developed originally by Philip van Damme. She put it to work on blood samples from a large Belgian founder family whose 43 patients showcase the dramatic clinical heterogeneity of progranulin mutations. Their clinical diagnoses range from $\mathrm{FTD}, \mathrm{AD}, \mathrm{PD}$, primary progressive aphasia (PPA), and progressive non-fluent aphasia (PNFA) all from having inherited the same mutation. Pathologically, this family runs the gamut, too, with Lewy bodies, ubiquitin-positive FTLD-U inclusions, amyloid pathology, and of course TDP-43.

From this family, Sleegers had serum of six patients, eight younger still-unaffected mutation carriers, and nine non-carriers. The ELISA distinguished carriers and non-carriers unequivocally, Sleegers showed. The groups were completely separate and apart by a large distance. Interestingly, the non-symptomatic carriers had the same progranulin levels as their affected relatives, suggesting the ELISA may be able to detect preclinical disease and presymptomatic mutation carriers. Genetic testing can do this, too, but it is more complicated to interpret, as scientists need to know whether a change in the gene sequence is pathogenic or a harmless variation, and genetic deletions require further analysis. Besides capturing all types of progranulin mutation, a blood-based ELISA could also be cheaper than genetic testing.

In her talk, Rosa Rademakers of the Mayo Clinic Jacksonville, Florida, reported the same results in a different, larger group of patients. Rademakers is a neurogeneticist also formerly of van Broeckhoven's group; she received a Young Investigator Award at the conference. Her team optimized a commercial ELISA for human progranulin and tested plasma of 219 patients clinically diagnosed with FTD. In this study, too, all patients carrying a loss-of-function progranulin mutation had only about one-third as much progranulin in their blood as did patients without a progranulin mutation. The ELISA predicted with 100 percent certainty that everyone with less than 112 nanogram/milliliter (ng/ml) of the growth factor carried a progranulin mutation. This is nearly identical to the cutoff suggested in an earlier Italian study led by Giuliano Binetti at the Centro San Giovanni di Dio-Fatebenefratelli in Brescia, which tested plasma and CSF ELISAs in a group of FTLD patients.

Working in parallel, both groups next studied whether their ELISAs could tease apart some of the multiple disease processes underlying a clinically defined disease, in other words, serve as a new tool to better define the spectrum of neurodegeneration. For example, Sleegers reported that progranulin was low in the serum of a person who had been diagnosed with AD but later proved to have a loss-of-function progranulin mutation. Conversely, Rademakers showed that the plasma test revealed abnormally low progranulin in one of 72 people clinically diagnosed with AD and that this man, upon sequencing, proved to have a new lossof-function progranulin mutation. Likewise, a French man with clinical Parkinson disease and a progranulin mutation also had low plasma progranulin. "Regardless of how a person presents clinically, the ELISA detects a progranulin null mutation," Sleegers said.

Lastly, both studies explored whether their ELISAs were able to pick up more subtle genetic flaws in progranulin. For example, missense mutations cause less than haploinsufficiency, which results from a mutation that aborts protein production entirely. Researchers are exploring different kinds of missense mutations in this gene. Some hasten the degradation of the protein, others reduce its secretion, and in-silico modeling points to misfolding at the protein's internal disulfide bridges as a possible cause for these cellular problems with the protein. In Prague, Sleegers closed her talk with data showing that both in people with clinical FDT and AD, missense mutations that such research had predicted to be pathogenic came with reduced serum progranulin levels in their carriers, though the drop was less precipitous than with a null mutation. Missense mutations predicted to be harmless corresponded to normal levels of serum progranulin.

Last but not least, also in Prague, German researchers led by Anja Capell and Christian Haass at LudwigMaximilian University in Munich presented ongoing work on a third ELISA to quantify progranulin in the CSF. Compared to serum and plasma, where progran- 
ulin levels ranged in the low hundreds of $\mathrm{ng} / \mathrm{ml}$ for controls and 50 to $90 \mathrm{ng} / \mathrm{ml}$ in null mutation carriers, CSF levels are much lower, around 5-7 $\mathrm{ng} / \mathrm{ml}$ in controls. Previous work has reported this same range in controls and about $2 \mathrm{ng} / \mathrm{ml}$ in mutation carriers. In clinical practice, it is not clear if a spinal tap will be necessary eventually, because blood-based tests appear to work well so far, Rademakers wrote by E-mail.

All told, these studies suggest that blood tests could reveal an underlying progranulin-driven disease process regardless of how it manifests clinically. It is simpler than genetics because it picks up the loss of the protein no matter which of a myriad of different genetic changes might be to blame. Such a blood test could show whose early-onset dementia is due to this particular protein, and predict future neurodegeneration in people who are still cognitively healthy but at risk because of their family history. Viewed broadly beyond FTD, progranulin tests could help explain a slice of the neurodegenerative disease spectrum.

\section{RESHUFFLE PARKINSON'S GENETICS TO LAY OUT ITS PATHWAYS?}

9 June 2009. This penultimate article in the Alzforum series on the spectrum of overlapping neurodegenerative diseases reports the call of a founding neurogeneticist, who rocks the boat from time to time, to reorganize the genetics of Parkinson's and related diseases. In a series of provocative talks, including one at a workshop on Dementia with Lewy Bodies and Parkinson's Disease Dementia held last March in the German city of Kassel, and again in a plenary thereafter at the 9th International conference AD/PD 2009 in Prague, John Hardy of University College London, UK, called on his audiences to cast a critical eye over the current list of proposed Parkinson's genes and engage in some spring cleaning. Out with some genes, in with others, and the field could benefit by seeing - voila - a common metabolic pathway for Lewy body disorders, Hardy claimed.

In essence, the idea is that if three genes currently regarded as PD genes were instead set aside as genes for a mitochondrial path to neuronal death in the substantia nigra, and if other genes known to cause clinically different diseases that nonetheless come with Lewy body pathology were included, then the field might make faster progress in understanding molecular mechanisms of Lewy body diseases. That's because scientists would have a list of genes that all impinge in some way on the metabolism of the brain glycolipid ceramide. Two papers out this month in Archives of Neurology on one such gene - glucocerebrosidase (GBA) - support this claim by placing GBA front and center as the strongest risk factor to date for sporadic PD and DLB.

With such a reorganization of the PARK loci, Hardy said, scientists would avoid being misled by a bewildering variety of clinical parkinsonian phenotypes. Rather than following clinical descriptions, gene sleuths could instead grab hold of the pathology as a starting point to find the underlying gene mutations and risk variants that drive pathogenesis. In a review article published this month, Hardy and colleagues recall that basing genetics on pathology has served Alzheimer disease research well. Starting with Alois Alzheimer, AD was defined from the get-go by its definitive pathology of plaques and tangles. This helped geneticists find the autosomal-dominant genes, and the molecular biology of AD took off as a field when those three genes turned out to fall into the very pathway of APP metabolism that George Glenner had anticipated with his biochemical isolation of amyloid from brain. Other clinical dementias - e.g., vascular, frontotemporal - were declared different and thus did not distract the geneticists. "Just think if we had gone for genes for dementia, not for Alzheimer disease. We would be in a terrible mess," Hardy wrote to ARF.

Parkinson disease has a different history. First, unlike Alzheimer's 1906 paper, James Parkinson's eponymous description, in 1817 , of the shaking palsy was clinical. Second, the breakthrough of L-Dopa therapy in the 1960s rightly reinforced the importance of recognizing the clinical features because patients responded so well to this symptomatic drug, the scientists write in their review. Later it became clear that most people with PD have Lewy bodies. But so do people who suffer from other clinical diseases, and the pathology in PD generally took a back seat to the clinical perspective. While clinical classification is fine for treatment, however, it can lead molecular pathogenesis research into a thicket and delay the delineation of the multiple protein-driven pathways that give rise, either alone or in combination, to a person's individual combination of clinical symptoms, Hardy claims.

Such a reassessment is timely now. It would cap a decade during which genetics has risen to prominence in PD research. Before that, Parkinson's was widely considered a sporadic disease, but the initial discovery in 1997 of an a-synuclein mutation in a Greek/Italian family with early-onset PD triggered a period of genetic inquiry that currently amounts 


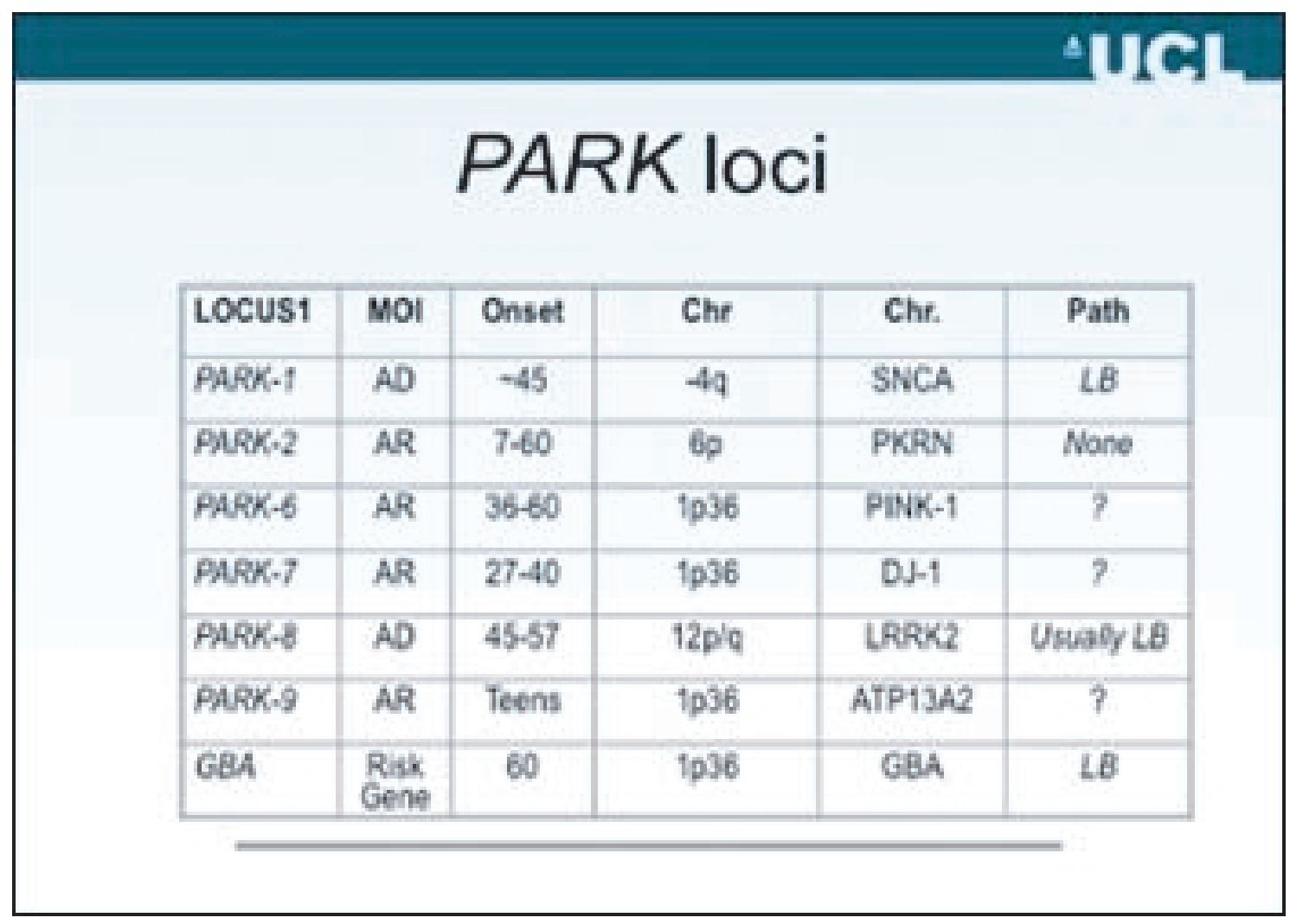

Fig. 1.

to 457 genes being tracked in the PDGene database (http://www.pdgene.org/).

Figure 1 shows a current selection of the best-known PARK loci Hardy showed in Prague.

What does he find wrong with this picture? The right column shows that three well-known genes generally cause no Lewy body pathology or at least have not been reported as such yet. Parkin, Pink-1, and DJ-1 are inherited in an autosomal-recessive mode, and generally come as loss-of-function alleles. To the geneticist, proof of pathogenicity for some of those, particularly missense alleles, can be difficult to establish. Yet the primary reason Hardy suggests for grouping these three separately is that they appear to act in the same underlying pathway that is separate from the pathway of $\alpha$-synuclein, LRRK2, and the new slugger in PD/DLB genetics, GBA (see part 9). Pink-1 acts upstream of parkin in the same mitochondrial pathway; DJ-1, while not directly placed in the same pathway, is an essential part of the oxidative stress response in mitochondria, too. Clinically, mutations in these three genes tend to give rise to very early-onset syndromes of juvenile parkinsonism starting, in rare cases, even in child- hood. In essence, Hardy suggests this: these genes are important, but they cause a mitochondrial path to parkinsonian symptoms that is probably separate from a main PD/DLB pathway that gives rise to the predominant pathology of intracellular $\alpha$-synuclein aggregates. Hence, Hardy moves them to the left in his suggested pathway diagram below:

View larger image (http://www.alzforum.org/images /new/pathway_lg.jpg).

With the 3 green mitochondrial genes on the left, what's happening on the right? Hardy suggested that the protein aggregation that leads to Lewy bodies and Lewy neurites (the main types of $\alpha$-synuclein pathology visible to microcopy) encompasses a set of genes that are not typically seen as belonging to the PD spectrum, but deserve consideration. First, three smaller ones, then the big one. The little-known PLA2G6 gene causes an adult-onset parkinsonian movement disorder with cognitive and psychiatric symptoms, and the PANK2 gene causes a severe movement disorder with mental deterioration called Hallervorden-Spatz. Importantly, both of these rare diseases have Lewy pathology. Their proteins both also happen to map to different 


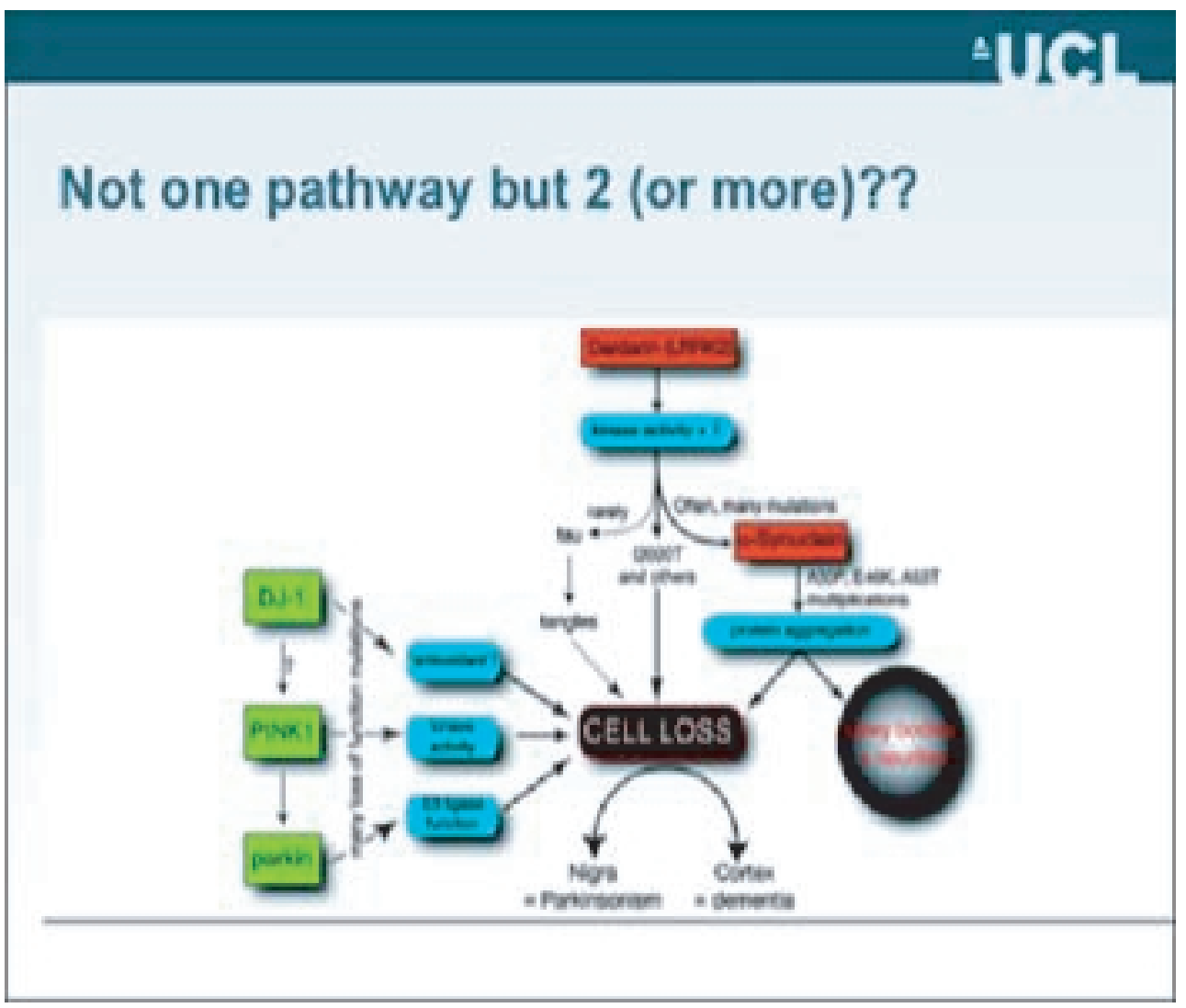

Fig. 2.

arms of lysosomal ceramide metabolism. When they function normally, they reduce levels of this lipid (see diagram below by Jose Bras in Hardy's group).

View larger image (http://www.alzforum.org/images /new/ceramide Jg.jpg).

Then there is the better-known Niemann-Pick Type C (NPC) gene. Recessive mutations cause the childhoodonset lysosomal storage disease known for its progressive neurodegeneration, though its phenotype varies widely. This disease might fit in because it also features Lewy bodies, and the NPC mutant from patients is known to reduce the activity of acid sphingomyelinase, an enzyme in this pathway, Hardy writes in the review. The physiological function of $\alpha$-synuclein, the founding member of Lewy body disease genetics and the protein at the heart of its pathology, is still largely a mystery. But besides its synaptic localization, a biochemical role in brain lipid metabolism is likely, Hardy said.

The big new anchor for this scenario, however, is GBA, the gene for the enzyme glucocerebrosidase. Five new papers published over the last two months alone strengthen its central role in the genetics of Lewy body diseases.

\section{MORE THAN GAUCHER'S - GBA THROWS ITS WEIGHT AROUND LEWY BODY DISEASE}

9 June 2009. This news story on the glucocerebrosidase (GBA) gene closes the Alzforum series on emerging concepts in the neurodegenerative disease spectrum. This latest genetics discovery may help scientists distinguish cases on the Lewy body end of the spectrum (i.e., toward PD and some dementia with Lewy bodies) from the plaque and tangle end of the spectrum (i.e., AD). This year alone has seen five original research papers and several reviews on the growing realization that heterozygous mutations and pathogenic alleles of this enzyme are the most prevalent risk factor known to date for PD and other Lewy body diseases. "This is a big story," said John Hardy, who argued at recent conferences that the GBA gene deserves a central place in the genetic lineup of Lewy 


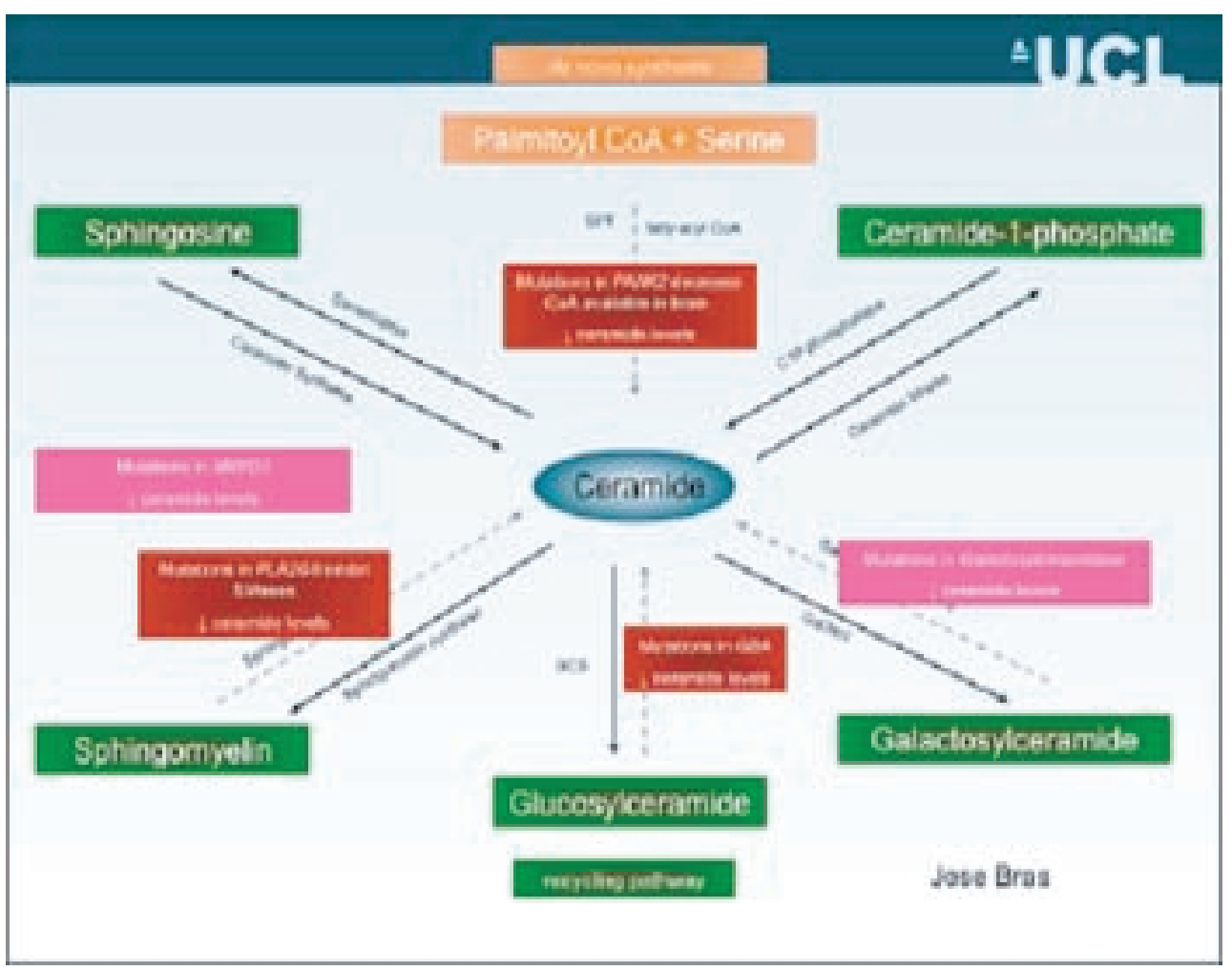

Fig. 3.

body disease genes. GBA already ranks first on the Top PDGene Results (http://www.pdgene.org/). Even since that database was last updated, a torrent of new data on larger patient groups and a greater number of rare disease variants appeared online this month. It further solidifies the position of the Gaucher disease gene as a major risk gene for sporadic and even familial clustering of these diseases. "We can have confidence that GBA has an important role in the pathogenesis of Lewy body disorders," wrote James Leverenz of University of Washington, Seattle, and colleagues in an editorial accompanying the latest two papers in Archives of Neurology.

Until recently, GBA was known primarily for causing Gaucher disease. This is an autosomal recessive, lysosomal storage disease of glucocerebrosidase deficiency. It can cause acute liver damage even early in life, typically in homozygous mutation carriers, when the substrate of the mutated, sluggish enzyme - the lipid glucocerebroside - accumulates in cells. At some 10,000 estimated cases worldwide, Gaucher's is an orphan disease. The gradual expansion of GBA's relevance to a much larger group of people historically began with case reports of parkinsonism in Ashkenazi
Jewish patients with Gaucher disease. These reports initially drew little attention among PD epidemiologists and geneticists, said Hardy. More widely noted, at least in the U.S., were papers by Ellen Sidransky at the NIH in Bethesda, Maryland, who pursued her clinical observation that fathers and uncles of her patients with Gaucher's tended to show parkinsonian symptoms. Sidransky's group conducted a series of small studies looking at neuropathology and GBA mutations in family members.

Together, the Israeli and U.S. work inspired groups worldwide to look for GBA mutations in PD patient series of non-Ashkenazi origin. These studies were generally small and only assessed specific GBA SNPs known from Gaucher disease, not the entire sequence of the gene, but even so, many of them were positive. Overall, this existing work led to a sense that the acute liver problems of Gaucher's develop when a person lacks at least 80 percent of GBA activity, whereas milder, or the more common heterozygous mutations that eliminate about half of the body's GBA activity enable a healthy childhood but can cause later-onset Lewy body diseases such as PD or dementia with Lewy bodies, Hardy and colleagues write in a review out this month. 
In the past three months, the story suddenly bulked up when data on larger patient series pouring in. In Prague, Laura Parkkinen of University College London, UK, presented that group's latest results on 790 patients with PD and 257 controls. Four percent of the patients had one of 14 different GBA mutations, adding up to a total odds ratio of 3.7. All GBA carriers who had consented to autopsy showed extensive Lewy body pathology in their brain. Clinically, about half had typical PD; the other half also had the hallucinations and cognitive decline that marks dementia with Lewy bodies (DLB, see part 3 of this series). "GBA mutations are the most common genetic risk factor for developing sporadic PD or DLB in this large British population," Parkkinen said. This data appeared last March, as did the results of a separate series of 172 Greek Parkinson's patients in whom GBA also proved the most commonly mutated gene, amounting to a similar odds ratio of 4.2.

Still-larger datasets rolled in this month from Japan and New York. Led by Shoji Tsuji at University of Tokyo Graduate School of Medicine, first author Jun Mitsui and collaborators at other Japanese institutions reported that their re-sequencing effort of the entire GBA gene in 534 PD patients and 544 controls discovered 11 pathogenic variants that together occurred in a total of 10 percent of patients but in almost no controls, leading to a whopping odds ratio of 28 . The British, the Greek, and the Japanese studies, as had some previous ones, all found that GBA mutations showed up particularly in early-onset patients. The large patient group in the Japanese sample included 34 families with clusters of PD cases; of those families, eight had heterozygous GBA variants that showed up in all affected relatives, making GBA a gene not only for sporadic PD, but also for some forms of autosomal-recessive familial PD.

These data imply that the field will have to abandon the comparatively simple idea that common diseases like PD are caused by common gene variants. Instead geneticists are coming to grips with the more complex notion that common diseases are caused by many different variants, many of which will be rare. "We should emphasize a paradigm shift from the common disease common variants hypothesis to the common disease - multiple rare variants hypothesis in our search for disease susceptibility genes in sporadic PD, which may be applicable to studies of other diseases," Mitsui and colleagues wrote. In practice, this paradigm shift amounts a tall order, as finding these multiple rare variants requires extensive sequencing of the entire gene in cases as well as in many controls. For GBA, this is difficult because the existence of an adjacent pseudogene makes this whole genomic region challenging to dissect.

For its part, the New York City study, led by Karen Marder at Columbia University, focused on patients with dementia with Lewy bodies (DLB). Of 95 people who had pathologically confirmed Lewy body disease, fully 28 percent had GBA mutations. Relatively fewer, that is, 10 percent, of people who had AD pathology also had GBA mutations, as did 3 percent of controls without either AD or Lewy body pathology. These authors report that in their patient sample, GBA mutations tended to lead to extensive $\alpha$-synuclein pathology in the cortex. They suggest that in this way, GBA might serve as a diagnostic marker during life, indicating that mutation carriers likely have "purer" Lewy body pathology and that their dementia results primarily from that, not from the amyloid and tau pathology that marks AD.

How GBA variants cause PD and DLB is unknown at this point, because the molecular work studying the variants remains to be done. However, in these early days, most scientists interviewed for this article leaned toward a loss-of function mechanism. Some scientists noted that research into whether glucocerebrosidase variants might impair protein degradation in lysosomes might lead to new insights about $\alpha$-synuclein processing and aggregation. Others raised the notion that GBA enzyme activity could provide a basis for developing fluid biomarkers, similarly to the way they are coming along for progranulin, a growth factor whose own loss of function is genetically linked to many cases of frontotemporal dementia. That is in the future; but even now, scientists agree that recent genetics news have noticeably shifted the PD and DLB landscape toward lysosomal ceramide metabolism as a promising area of research. 